\title{
The feasibility and safety of radical esophagectomy in patients receiving neoadjuvant chemoradiotherapy with pembrolizumab for esophageal squamous cell carcinoma
}

\author{
Seong Yong Park ${ }^{1 \#}$, Min Hee Hong ${ }^{2 \#}$, Hye Ryun $\mathrm{Kim}^{2}$, Chang Geol Lee ${ }^{3}$, Jae Ho Cho ${ }^{3}$, \\ Byoung Chul $\mathrm{Cho}^{2}$, Dae Joon Kim ${ }^{1}$ \\ ${ }^{1}$ Department of Thoracic and Cardiovascular Surgery, Yonsei University College of Medicine, Seoul, Republic of Korea; ${ }^{2}$ Division of Medical \\ Oncology, Department of Internal Medicine, Yonsei Cancer Center, Severance Hospital, Yonsei University College of Medicine, Seoul, Republic of \\ Korea; ${ }^{3}$ Department of Radiation Oncology, Yonsei University College of Medicine, Seoul, Republic of Korea \\ Contributions: (I) Conception and design: SY Park; (II) Administrative support: DJ Kim, BC Cho; (III) Provision of study materials or patients: BC \\ Cho, MH Hong, HR Kim; (IV) Collection and assembly of data: SY Park, MH Hong; (V) Data analysis and interpretation: SY Park, MH Hong; (VI) \\ Manuscript writing: All authors; (VII) Final approval of manuscript: All authors. \\ \#These authors contributed equally to this work as co-first authors. \\ Correspondence to: Dae Joon Kim. Department of Thoracic and Cardiovascular Surgery, Yonsei University College of Medicine, 50 Yonsei-ro, \\ Seodaemun-gu, Seoul 120-752, Republic of Korea. Email: kdjcool@yuhs.ac.
}

Background: Immune checkpoint inhibitors have been tried for several thoracic malignancies; however, their application as a neoadjuvant therapy in esophageal squamous cell carcinoma (ESCC) has not been studied. We evaluated the feasibility and safety of esophagectomy and total lymphadenectomy after neoadjuvant chemoradiation therapy with pembrolizumab.

Methods: Between 2017 and 2018, 38 patients who received the neoadjuvant therapy followed by radical esophagectomy and total lymphadenectomy for ESCC were analyzed. Twenty-two patients received conventional neoadjuvant chemoradiation therapy (Group 1), and sixteen patients received neoadjuvant chemoradiation therapy with pembrolizumab in clinical trial (Group 2). Two groups were compared retrospectively.

Results: The basic characteristics of age, clinical stage, location and methods of operation were not different between the two groups. The pathologic stages were higher in Group 2, but the difference was not statistically significant. The operative outcomes, i.e., operation time, blood loss, and numbers of dissected lymph nodes in the thorax, neck, and abdomen were comparable. Complications, including pulmonary complications and anastomotic leakage, were also comparable. The rate of recurrent laryngeal nerve palsy was also comparable between the two groups $(31.8 \%$ vs. $18.8 \%, \mathrm{P}=0.469)$. Operative mortalities developed in 2 patients [0 vs. 2 (12.5\%), $\mathrm{P}=0.171$ ] due to acute respiratory distress syndrome (ARDS).

Conclusions: Radical esophagectomy for esophageal squamous cell carcinoma after neoadjuvant chemoradiation therapy with pembrolizumab may not increase the operative risk or reduce the quality of radical dissection including lymphadenectomy. The risk of ARDS after neoadjuvant neoadjuvant chemoradiation therapy with pembrolizumab has to be studied in the further analysis.

Keywords: Esophagectomy; immune checkpoint inhibitors; neoadjuvant therapy; operative outcomes

Submitted Feb 29, 2020. Accepted for publication Sep 04, 2020.

doi: $10.21037 /$ jtd-20-1088

View this article at: http://dx.doi.org/10.21037/jtd-20-1088

^ ORCID: 0000-0002-5180-3853. 


\section{Introduction}

The mainstay of treatment for esophageal squamous cell carcinoma (ESCC) includes surgical treatment, chemotherapy, and radiotherapy, and it has been reported that multidisciplinary treatment combining these three modalities is most effective (1). Although the improvement of surgical technique and chemoradiation (CCRTx) strategies for ESCC have been achieved, the survival of esophageal cancer after multimodality therapy and esophagectomy still remains low; the 5 -year overall survival of ypStage I and ypStage II are about $50 \%$ and $30 \%$, respectively, in the Worldwide Esophageal Cancer Collaboration (WECC) data (2). Therefore, developing novel therapeutic strategies in addition to the conventional strategies is urgently needed to improve long-term survival.

Recent breakthrough results from immune checkpoint inhibitors such as an anti-cytotoxic T-lymphocyteassociated protein 4 (CTLA4) antibody (ipilimumab) and anti-programmed death 1 (PD-1) antibody have paved the way for a new era of cancer immunotherapy, leading to paradigm shift in cancer treatment (3). In ESCC, the results of the clinical trials using anti-PD1 antibody (nivolumab and pembrolizumab) in advanced stage were promising (4). Because the immunotherapy for the other thoracic malignancies, such as non-small cell lung cancer, have been studied extensively not only for the advanced and unresectable stages but also for the neoadjuvant setting in resectable disease, immunotherapy for ESCC could also be tried in the neoadjuvant setting. In addition, perioperative safety and the risk of serious adverse events after esophagectomy have not been adequately addressed even though the number of indications and patients on trials being treated with immunotherapy has increased. Especially, esophagectomy is an invasive procedure in oncologic surgery with high morbidities and mortalities, and there are no previous studies evaluating the safety and feasibility of radical esophagectomy after neoadjuvant immunotherapy followed by esophagectomy. Therefore, the purpose of this retrospective study was to evaluate the safety and feasibility of radical esophagectomy after neoadjuvant immunotherapy. We present the following article in accordance with the STROBE reporting checklist (available at http://dx.doi.org/10.21037/jtd-201088).

\section{Methods}

\section{Study data and patient selection}

This study conformed to the provisions of the Declaration of Helsinki (as revised in 2013). This retrospective study was approved by the Institutional Review Board (20191950-001) and the informed consents were waived by approval of Institutional Review Board. Between 2017 and 2018, 38 patients at our institution received neoadjuvant therapy followed by radical esophagectomy and total lymphadenectomy for ESCC. The indication of neoadjuvant therapy for ESCC in our institution was clinical stage T1N1-2 or T2-4aN0-2 (AJCC 7 TNM classification). Among them, the patients who received conventional neoadjuvant chemoradiation therapy were assigned as Group 1, whereas the patients who received neoadjuvant chemoradiation therapy pembrolizumab were assigned as Group 2 (Figure 1). Neoadjuvant chemoradiation therapy with pembrolizumab was conducted as the single-arm, prospective phase II trial (Clinicaltrials.gov identifier: NCT02844075). Because the clinical trial was single-arm study, we retrospectively compared patients in Group 2 with patients receiving conventional neoadjuvant CCRTx (Group 1) during the same study period. The inclusion criteria included in both groups were the same: (I) histologically or cytologically confirmed ESCC, (II) age 220, (III) ECOG PS: 0-1, (IV) clinical stage T1N1-2 or T2-4aN0-2 (AJCC 7 TNM classification), (V) be willing to provide tissue from a newly obtained core or excisional biopsy of a tumor lesion through repeated biopsies, and (VI) adequate organ function.

\section{Regimen of neoadjuvant therapy}

Patients enrolled in Groups 1 and 2 received a total radiation dose of 44.1 Gy with 21 fractions and 5 fractions per week. The treatment in Group 1 consisted of continuous intravenous 5 -fluorouracil $1,000 \mathrm{mg} / \mathrm{body}-$ surface area (BSA) for 4 days, and cisplatin $75 \mathrm{mg} / \mathrm{BSA}$ for 1 day every 4 weeks for two cycles in neoadjuvant period. The treatment in Group 2 included paclitaxel, carboplatin, and pembrolizumab. Paclitaxel at a dose of $45 \mathrm{mg} / \mathrm{BSA}$ and carboplatin targeted at an area under the curve of $2 \mathrm{mg}$ per milliliter per minute were administered weekly for a total of 5 doses along with pembrolizumab $200 \mathrm{mg}$ every 3 weeks (total 2 doses). 


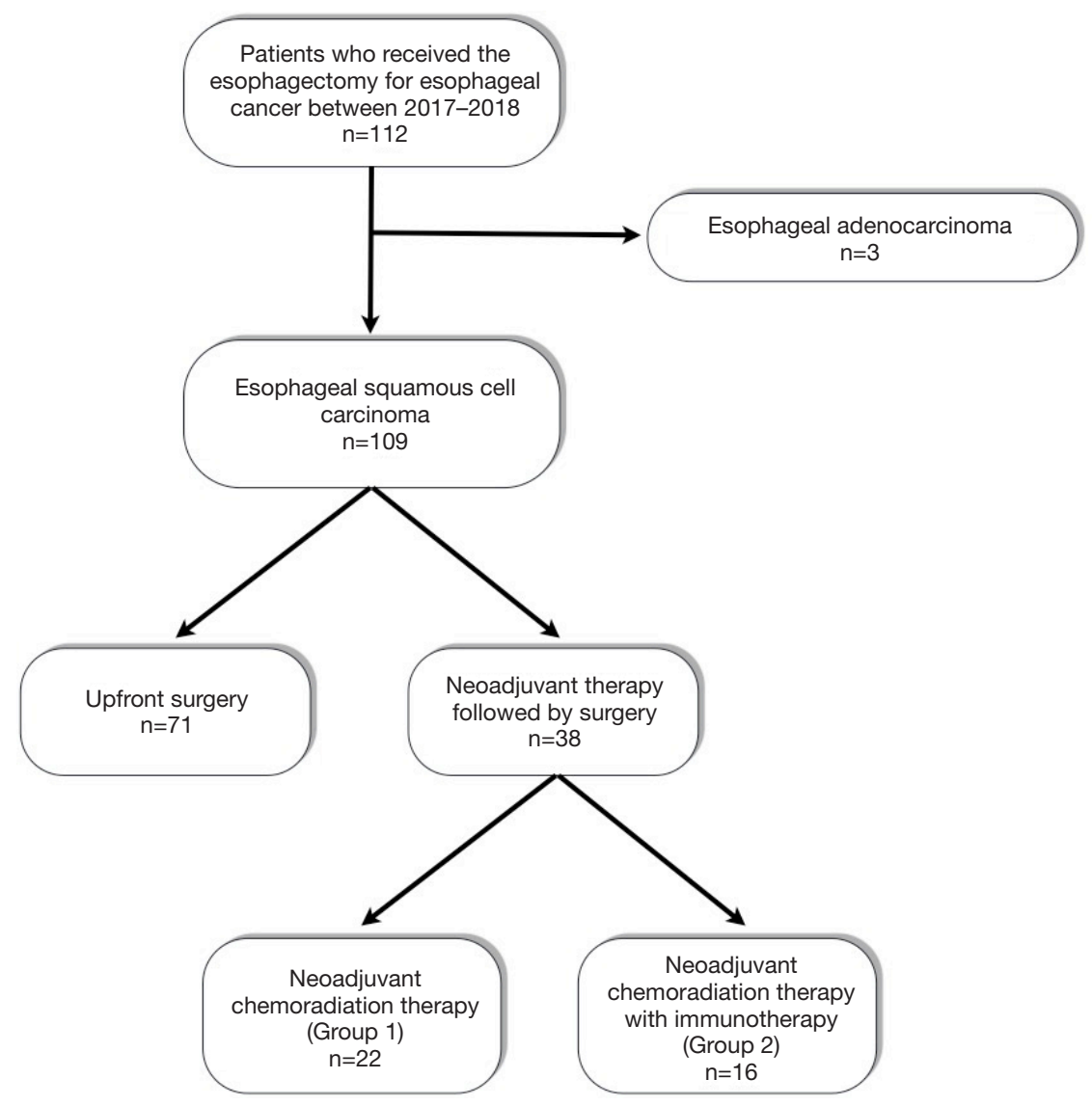

Figure 1 Characteristics of patients enrolled in this study.

\section{Operation}

The operation was done between 6 to 8 weeks after neoadjuvant therapy. Esophagectomy and total lymphadenectomy were done in all patients. Regarding the thoracic phase, minimally invasive esophagectomy, such as thoracoscopic or robotic esophagectomy, was the treatment of choice, but open thoracotomy was also performed in cases of T3 lesions or suspicious T4 lesions. Total lymphadenectomy, the complete dissection of the bilateral recurrent laryngeal nerve nodes, was performed in all patients. Neck dissection was performed when metastatic lymph nodes were suspected at the cervical area. Esophageal reconstruction was done with gastric tube and cervical anastomosis. After operation, the operating surgeon dissected the lymph nodes before sending the specimen to the pathologic department.

\section{Definition of complications and surgical outcomes}

Postoperative complications were defined based on the
Esophageal Complications Consensus Group (ECCG) criteria (5). In the ECCG criteria, pneumonia is defined as the presence of a new or progressive radiographic infiltrate plus at least two of three following clinical features: (I) fever greater than $38^{\circ} \mathrm{C}$, (II) leukocytosis or leukopenia, or (III) purulent secretions. Acute respiratory distress syndrome (ARDS) was defined based on the Berlin definition (6). Vocal cords were routinely evaluated by the otolaryngologist to detect vocal cord palsy at postoperative day (POD) 7 . The esophagography was done at POD 7 to evaluate the anastomotic integrity. The $\mathrm{R} 0$ resection was defined as negative resection margin as no cancer cells at the proximal, distal and circumferential margin.

\section{Statistical analyses}

All observed data are presented as medians and interquartile range deviations for continuous variables and as frequencies and percentages for categorical variables. Groups 1 and 2 
Table 1 Patient basic characteristics

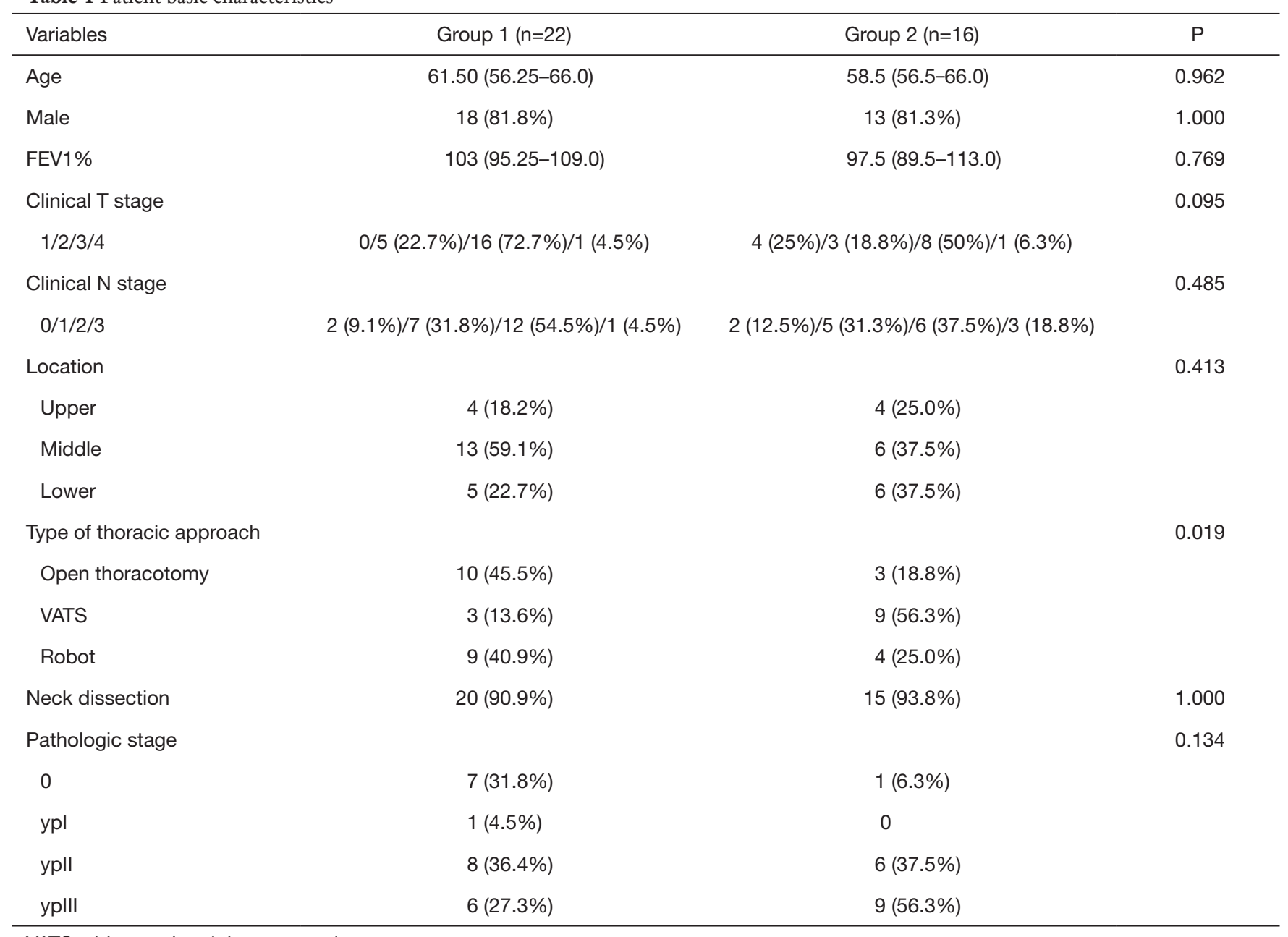

VATS, video-assisted thoracoscopic surgery

were compared by the Kruskal-Wallis test or independent $t$-test for continuous variables according to the normality of the data and by the chi-square test or Fisher's exact test for categorical variables. A two-sided $\mathrm{P}$ value of 0.05 was considered to indicate a statistically significant difference for all analyses. All statistical procedures were performed using $\mathrm{R}$ version 2.15.1 (R Foundation for Statistical Computing, Vienna, Austria).

\section{Results}

\section{Patient and operative characteristics}

Among 38 patients, 22 patients received conventional neoadjuvant chemoradiation therapy (Group 1), whereas 16 patients received neoadjuvant chemoradiation therapy with pembrolizumab (Group 2, Figure 1). Basic clinical characteristics are described in Table 1. The mean age and sex were not different between the two groups. Open thoracotomy was more frequently performed in Group 1 ( $45.5 \%$ vs. $18.8 \%, \mathrm{P}=0.019)$. However, there were no thoracotomy conversion during the thoracoscopic and robotic esophagectomy in either group. Other variables such as clinical stage and neck dissection were not different between the two groups. Group 2 showed a higher ypStage pathologic stage than Group 1, but the difference was not statistically significant.

\section{The incidence of immune-related complications in Group 2}

There were 1 hypothyroidism (grade 1) and 1 acneiform rash (grade 1) which were considered as immune-related 
Table 2 Operative outcomes

\begin{tabular}{|c|c|c|c|}
\hline Variables & Group $1(n=22)$ & Group $2(n=16)$ & $\mathrm{P}$ \\
\hline Blood loss (mL) & $225(150-362.5)$ & $225(162.5-430)$ & 0.420 \\
\hline Numbers of total dissected LNs & $53.5(31-69.25)$ & $57(45.25-74.25)$ & 0.205 \\
\hline Numbers of positive LNs & $1(0-2.25)$ & $2(1-4)$ & 0.085 \\
\hline Numbers of dissected LNs in Rt. RLNs & $3.5(1-5)$ & $3.5(1-4.75)$ & 0.535 \\
\hline Numbers of dissected LNs in Lt. RLNs & $2.5(1-6)$ & $2(1-5)$ & 0.491 \\
\hline Numbers of dissected LNs in neck & $12(8-25.25)$ & $18(13-28.5)$ & 0.130 \\
\hline Numbers of dissected LNs in abdomen & $12(8-21.25)$ & $17(11.25-20)$ & 0.807 \\
\hline
\end{tabular}

LN, lymph node; RLN, recurrent laryngeal nerve.

in neoadjuvant period. We observed these two patients without specific medication such as thyroid hormone replacement or corticosteroid. And despite the immunerelated adverse events, all subjects had undergone preplanned R0 resection. In the adjuvant period, there were 8 hepatitis (grade 1 or 2), one grade 1 hypothyroidism, and three grade 3 hypothyroidism. No serious adverse events or adverse events which led to treatment interruption or discontinuation occurred. Three subjects who experienced grade 3 hypothyroidism were managed with thyroid hormone replacement. All immune-related adverse events were manageable.

\section{Operative outcomes}

Regarding the postoperative outcomes, the operation time and blood loss were similar between the two groups (Table 2). The numbers of dissected lymph nodes were higher in Group 2 [53.5 (31-69.25) vs. 57 (4.25-74.25)], but differences were not statistically different $(\mathrm{P}=0.205)$. The numbers of dissected lymph nodes in neck, thorax, and abdomen were also similar. The numbers of left and right recurrent laryngeal nerve nodes were also comparable. The numbers of positive lymph nodes were higher in Group 2 than in Group 1 [1 (0-2.25) vs. 2 (1-4)], but they were statistically marginal $(\mathrm{P}=0.085)$. All patients received the $\mathrm{R} 0$ resection.

The operative complications are summarized in Table 3. The incidence of ARDS was higher in Group 2 than in Group 1 (18.8\% vs. $4.5 \%)$, but it was not statistically different $(\mathrm{P}=0.291)$. The incidences of other complications such as recurrent laryngeal nerve palsy and anastomotic leakage were also similar. All anastomotic leakage was ECCG grade II. In-hospital mortalities occurred in Group 2. The length of hospital stay was not statistically different between the two groups, but they tended to be longer in Group $2(22.45 \pm 15.09$ vs. $41.19 \pm 45.59$ days, $\mathrm{P}=0.132$ ).

Two cases of operative mortality occurred in Group 2. The first case was a 62 years old male patient. He underwent an open esophagectomy and total mediastinal lymph node dissection, three field lymph node dissection, and cervical esophagogastrostomy. At POD 2, bilateral infiltration developed in his lower lung field and ARDS was diagnosed based on the $\mathrm{PaO}_{2} / \mathrm{FiO}_{2}$ ratio. He did not recover from the ARDS and died at POD 146.

The second case was a 72 -year-old male patient with underlying pulmonary emphysema. He received a thoracoscopic esophagectomy and total mediastinal lymph node dissection, three field lymph node dissections, and a cervical esophagogastrostomy. At POD 6, bilateral infiltration was detected on chest X-ray, and a chest CT showed bilateral diffuse ground glass opacity and interlobar septal thickening (Figure $2 A, B$ ). He received medical treatment including steroid pulse therapy and empirical antibiotics, but he died at POD 51.

\section{Discussion}

Immune checkpoint inhibitors have recently been 
Table 3 Postoperative complications

\begin{tabular}{|c|c|c|c|}
\hline Variables & Group $1(n=22)$ & Group $2(n=16)$ & $P$ \\
\hline Acute respiratory distress syndrome & $1(4.5 \%)$ & $3(18.8 \%)$ & 0.291 \\
\hline Recurrent laryngeal nerve palsy & $7(31.8 \%)$ & $3(18.8 \%)$ & 0.469 \\
\hline Type I & $5(22.8 \%)$ & $3(18.8 \%)$ & \\
\hline Anastomotic leakage & $3(13.6 \%)$ & $3(18.8 \%)$ & 0.682 \\
\hline Type II & $2(9.1 \%)$ & $3(18.8 \%)$ & \\
\hline Type III & $1(4.5 \%)$ & 0 & \\
\hline Chyloperitoneum & $1(4.5 \%)$ & 0 & 1.000 \\
\hline lleus & $1(4.5 \%)$ & 0 & 1.000 \\
\hline Operative mortality & 0 & $2(12.5 \%)$ & 0.171 \\
\hline
\end{tabular}
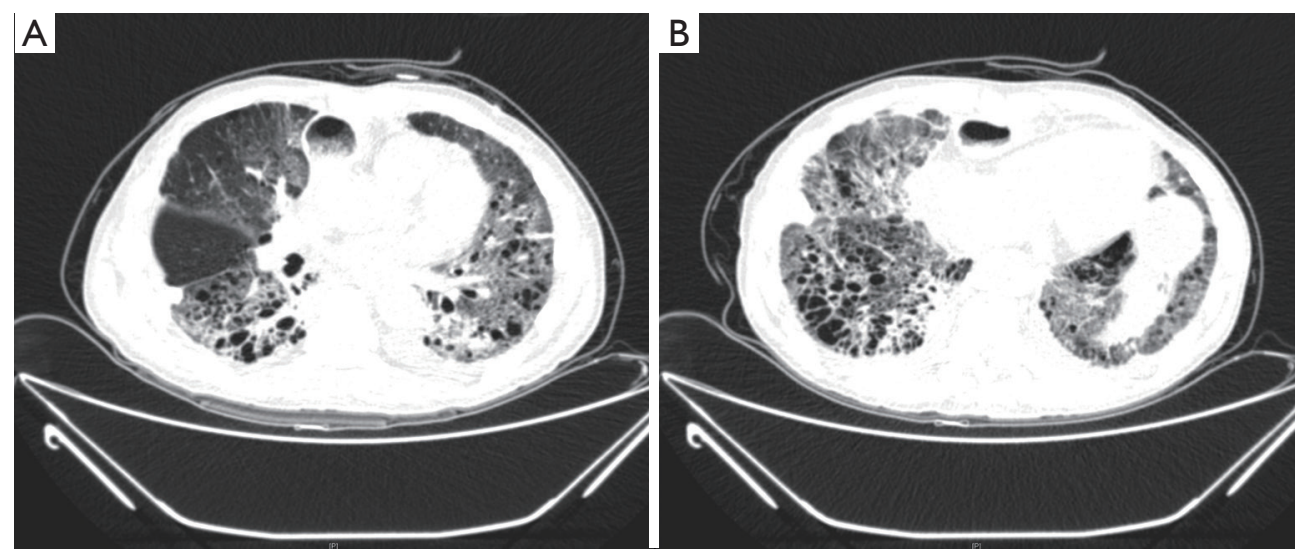

Figure 2 Chest CT findings of mortality case. The bilateral diffuse ground glass opacity and interlobar septal thickening developed in both lung fields. (A) Middle lung field. (B) Lower lung fields.

highlighted in the field of thoracic malignancy. The results from a phase II clinical trial of nivolumab for ESCC (4), in which 64 patients with ESCC who were refractory or intolerant to standard chemotherapy, including 5-fluorouracil, CDDP, and Taxan-based regimens, showed a complete or partial response in $14 \%$ of patients, and a disease control ratio was observed in $42 \%$. Moreover, the profile for adverse events was acceptable and manageable. Also, the KEYNOTE-181 study reported that pembrolizumab significantly improved OS compared with chemo as second-line therapy for advanced esophageal cancer with PD-L1 combined positive score $\geq 10$, accompanied by a more favorable safety profile (7). Based on these results, our group conducted a phase II trial of neoadjuvant CCRTx with pembrolizumab for ESCC; our results discussing the feasibility, safety and effectiveness of neoadjuvant therapy will be published separately. In the current paper, we focused on the operative outcomes for neoadjuvant CCRTx with Pembrolizumab, especially compared with conventional neoadjuvant CCRTx.

Neoadjuvant therapy has many potential advantages in esophageal cancer treatment. The CROSS trial showed the 

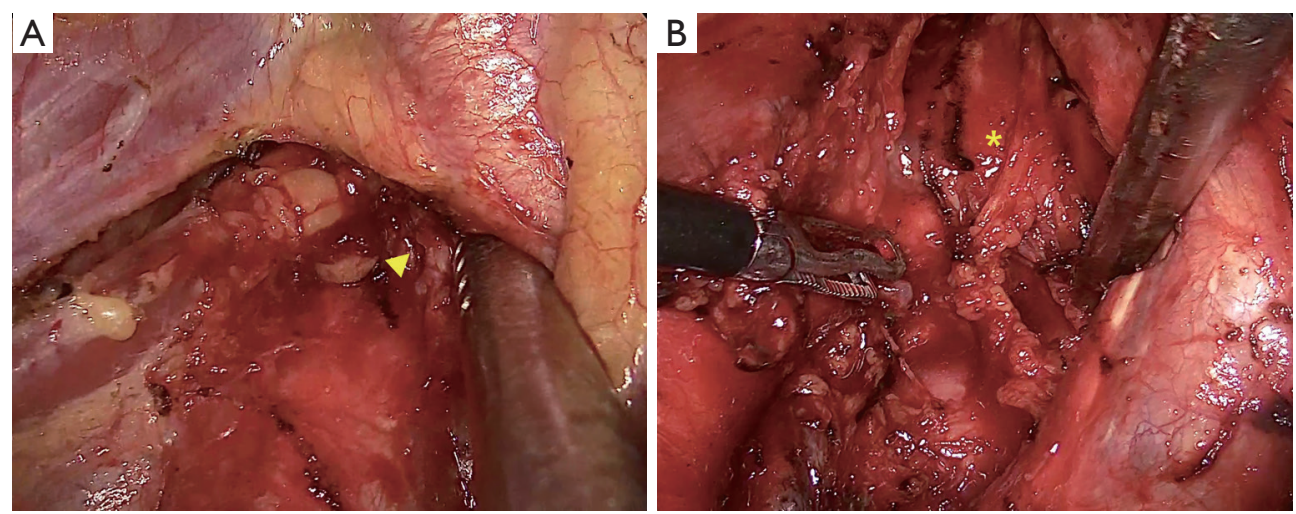

Figure 3 The radical dissection of bilateral recurrent laryngeal nerve lymph nodes after the neoadjuvant chemoradiation with immunotherapy. (A) Right recurrent laryngeal nerve lymph nodes. (B) Left recurrent laryngeal nerve lymph nodes.

overall survival benefits for neoadjuvant chemoradiotherapy when added to surgery in patients with resectable esophageal or esophagogastric junctional cancer, especially in ESCC (8). The JCOG 9907 trial determined that preoperative chemotherapy with cisplatin plus 5 -fluorouracil followed by surgery showed survival benefits compared with surgery followed by adjuvant therapy (9). In the viewpoint of surgeons, one of the most important concerns regarding neoadjuvant therapy is that the neoadjuvant therapy itself should not worsen the quality and surgical outcomes of any given operation. After preoperative immunotherapy, fibrosis around the tumor and tumor microenvironment are frequently reported (10). The initial retrospective case series in patients who received pulmonary resection for previously unresectable or metastatic non-small cell lung cancer suggest overall feasibility but have also raised concerns about technical challenges and perioperative issues unique to this patient population (10). Especially the radical esophagectomy including the dissection along the bilateral recurrent laryngeal nerve is the mainstay of treatment in ESCC, and we think that meticulous dissection, especially along the recurrent laryngeal nerve nodes, is an important procedure even after neoadjuvant therapy. Therefore, we were concerned that the quality of lymph node dissection might be decreased if immunotherapy was added in addition to CCRTx. In contrast to our initial assumption, dissection along the recurrent laryngeal nerve was feasible and safe in Group 2, and we could maintain our radical surgical strategies (Figure $3 A, B$ ). The numbers of dissected lymph nodes along the recurrent laryngeal nerve were comparable between two groups, and the incidence of vocal cord palsy, which can develop after recurrent laryngeal nerve dissection, was not statistically different between the two groups. These results imply that the preoperative immunotherapy did not decrease the quality of radial esophagectomy. In addition, the other major complication, anastomotic leakage, developed similarly in both groups. The longer hospital stays for Group 2 was might be related to the operative mortality cases, even though it was not statistically significant.

Even though it was not statistically significant, the incidence of ARDS was higher in Group 2 (18.8\% vs. 4.5\%, $\mathrm{P}=0.291$ ). Moreover, two patients showed operative mortality in Group 2. While the toxicity profile of immunotherapy is favorable compared with traditional cytotoxic therapy, the immune boosting mechanism of monoclonal antibodies can produce severe autoimmune adverse effects such as pneumonitis, arthralgia, pyrexia, colitis, dermatitis, hepatitis, endocrinopathy, and neuropathy (11). Large meta-analyses of randomized trials and retrospective studies using anti-PD1 and PDL1 antibodies across melanoma, lung cancer, and renal cancer found an overall incidence of pulmonary toxicity of $2.7-3.5 \%$ (12). It has been reported that the immune-related adverse events, including pulmonary toxicities, might occur at any point during treatment, and who will develop these toxicities or how long they will last is unclear (13). Pathologically, $80 \%$ of immune check-point inhibitor related pulmonary toxicities are T-lymphocytic alveolitis (13). Especially, it has been reported that the combination of radiotherapy with immunotherapy increased the risk of pulmonary toxicity when compared with the historical incidence of pulmonary toxicity with immunotherapy alone (14). In our series, two patients who received neoadjuvant CCRTx with 
immunotherapy died of pulmonary complications. It is possible that these pulmonary complications may be related to the immune check-point inhibitor associated pulmonary toxicities. However, acute lung injury also develops frequently after esophagectomy; the incidence of pulmonary complications including ARDS after esophagectomy in high-volume centers may exceed $20 \%$ (15). It is unclear whether the causes of ARDS in our patients were related to the immunotherapy or not. Therefore, further research is needed to determine whether the combination of CCRTx and immunotherapy is feasible, because of the potential synergistic effects of CCRTx and immunotherapy, especially after esophagectomy

In regard to immune-related adverse events, the median follow-up period of pembrolizumab arm was 18.3 months at the data cutoff and the incidence of immune-related hepatitis and hypothyroidism were $50 \%$ and $25 \%$, respectively and these numbers were higher compared to major clinical trials $(4,7)$. However, these events were all asymptomatic and the administration of pembrolizumab was not interrupted or delayed. Also, there were no serious adverse events and immune-suppressive treatment such as corticosteroid was not required at all. There was no new safety signal and the reported higher number of immunerelated adverse events may be attributable to the small sample size of the study.

This study has several limitations. First, this study is a retrospective study without randomization. Even though there was no statistical significance, Group 2 showed both higher clinical stage and higher pathologic stage (higher numbers of positive lymph nodes) outcomes. In Group 1, the esophagectomy under open thoracotomy was more frequently performed based on the surgeon's preferences. Second, the regimens of CCRTx were different between two groups. In South Korea, 5-fluororacil and cisplatin for esophageal cancer has been approved and reimbursed, but paclitaxel and carboplatin are not. Therefore Group 1 received infusional $5 \mathrm{FU} /$ cisplatin under the reimbursement and Group 2 patients could receive paclitaxel/carboplatin under the clinical trial. The purpose of this study was to compare the surgical outcomes, so the different CCRTx regimens might not be problematic. Third, the number of patients was small, so the further study has to be done with large numbers of patients with randomization. However, the we maintained uniform, radical surgical strategies in all patients. To our knowledge, this study is the first to report the surgical outcomes of neoadjuvant immunotherapy in ESCC. To overcome the limitations of this study, a randomized study with large numbers of patients is needed in the future.

In conclusion, radical esophagectomy for esophageal squamous cell carcinoma after neoadjuvant chemoradiation therapy with immune checkpoint inhibitors might not increase the operative risk or reduce the quality of radical dissection including lymphadenectomy.

\section{Acknowledgments}

Funding: This research was supported by Basic Science Research Program through the National Research Foundation of Korea (NRF) funded by the Ministry of Education (2019R1I1A1A01055513).

\section{Footnote}

Reporting Checklist: The authors have completed the STORBE reporting checklist. Available at http://dx.doi. org/10.21037/jtd-20-1088

Data Sharing Statement: Available at http://dx.doi. org/10.21037/jtd-20-1088

Conflicts of Interest: All authors have completed the ICMJE uniform disclosure form (available at http://dx.doi. org/10.21037/jtd-20-1088). The authors have no conflicts of interest to declare.

Ethical Statement: The authors are accountable for all aspects of the work in ensuring that questions related to the accuracy or integrity of any part of the work are appropriately investigated and resolved. The study conformed to the provisions of the Declaration of Helsinki (as revised in 2013). This retrospective study was approved by the Institutional Review Board (2019-1950-001) and the informed consents were waived by approval of Institutional Review Board.

Open Access Statement: This is an Open Access article distributed in accordance with the Creative Commons Attribution-NonCommercial-NoDerivs 4.0 International License (CC BY-NC-ND 4.0), which permits the noncommercial replication and distribution of the article with the strict proviso that no changes or edits are made and the original work is properly cited (including links to both the formal publication through the relevant DOI and the license). See: https://creativecommons.org/licenses/by-nc-nd/4.0/. 


\section{References}

1. Mariette C, Piessen G, Triboulet JP. Therapeutic strategies in oesophageal carcinoma: role of surgery and other modalities. Lancet Oncol 2007;8:545-53.

2. Rice TW, Ishwaran H, Kelsen DP, et al. Recommendations for neoadjuvant pathologic staging (ypTNM) of cancer of the esophagus and esophagogastric junction for the 8th edition AJCC/UICC staging manuals. Dis Esophagus 2016;29:906-12.

3. Kono K, Mimura K, Yamada R, et al. Current status of cancer immunotherapy for esophageal squamous cell carcinoma. Esophagus 2018;15:1-9.

4. Kudo T, Hamamoto Y, Kato K, et al. Nivolumab treatment for oesophageal squamous-cell carcinoma: an open-label, multicentre, phase 2 trial. Lancet Oncol 2017;18:631-9.

5. Low DE, Alderson D, Cecconello I, et al. International Consensus on Standardization of Data Collection for Complications Associated With Esophagectomy: Esophagectomy Complications Consensus Group (ECCG). Ann Surg 2015;262:286-94.

6. ARDS Definition Task Force; Ranieri VM, Rubenfeld GD, et al. Acute respiratory distress syndrome. JAMA 2012;307:2526-33.

7. Kojima T, Shah MA, Muro K, et al. Randomized Phase III KEYNOTE-181 Study of Pembrolizumab Versus Chemotherapy in Advanced Esophageal Cancer. J Clin Oncol 2020; JCO2001888.

8. Shapiro J, van Lanschot JJB, Hulshof MCCM, et al. Neoadjuvant chemoradiotherapy plus surgery versus

Cite this article as: Park SY, Hong MH, Kim HR, Lee CG, Cho JH, Cho BC, Kim DJ. The feasibility and safety of radical esophagectomy in patients receiving neoadjuvant chemoradiotherapy with pembrolizumab for esophageal squamous cell carcinoma. J Thorac Dis 2020;12(11):6426-6434. doi: $10.21037 /$ jtd-20-1088 surgery alone for oesophageal or junctional cancer (CROSS): long-term results of a randomised controlled trial. Lancet Oncol 2015;16:1090-8.

9. Ando N, Kato H, Igaki $\mathrm{H}$, et al. A randomized trial comparing postoperative adjuvant chemotherapy with cisplatin and 5 -fluorouracil versus preoperative chemotherapy for localized advanced squamous cell carcinoma of the thoracic esophagus (JCOG9907). Ann Surg Oncol 2012;19:68-74.

10. Bott MJ, Cools-Lartigue J, Tan KS, et al. Safety and Feasibility of Lung Resection After Immunotherapy for Metastatic or Unresectable Tumors. Ann Thorac Surg 2018;106:178-83.

11. Topalian SL, Hodi FS, Brahmer JR, et al. Safety, activity, and immune correlates of anti-PD-1 antibody in cancer. $\mathrm{N}$ Engl J Med 2012;366:2443-54.

12. Delaunay M, Cadranel J, Lusque A, et al. Immunecheckpoint inhibitors associated with interstitial lung disease in cancer patients. Eur Respir J 2017;50:1700050.

13. Rashdan S, Minna JD, Gerber DE. Diagnosis and management of pulmonary toxicity associated with cancer immunotherapy. Lancet Respir Med 2018;6:472-8.

14. Nishino M, Giobbie-Hurder A, Hatabu H, et al. Incidence of Programmed Cell Death 1 InhibitorRelated Pneumonitis in Patients With Advanced Cancer: A Systematic Review and Meta-analysis. JAMA Oncol 2016;2:1607-16.

15. Bailey SH, Bull DA, Harpole DH, et al. Outcomes after esophagectomy: a ten-year prospective cohort. Ann Thorac Surg 2003;75:217-22; discussion 222. 